\title{
Yod
}

Revue des études hébraïques et juives

La littérature israélienne, miroir d'une société multiple

\section{Le rapport judéo-arabe dans le roman de Abraham B. Yehoshua : La Mariée libérée}

The Jewish-Arab Relationship in the Novel by Abraham B. Yehoshua, The Liberated Wife ("La Mariée libérée")

יחסי יהודים-ערבים ברומן "הכלה המשוחררת" לא.ב.יהושע

\section{Françoise Saquer-Sabin}

\section{OpenEdition}

Journals

\section{Édition électronique}

URL : https://journals.openedition.org/yod/339

DOI : $10.4000 /$ yod.339

ISSN : 2261-0200

Éditeur

INALCO

\section{Édition imprimée}

Date de publication : 1 octobre 2009

Pagination : 67-91

ISBN : 978-2-85831-178-1

ISSN : 0338-9316

\section{Référence électronique}

Françoise Saquer-Sabin, « Le rapport judéo-arabe dans le roman de Abraham B. Yehoshua : La Mariée libérée ", Yod [En ligne], 14 | 2009, mis en ligne le 15 juin 2012, consulté le 08 juillet 2021. URL : http:// journals.openedition.org/yod/339; DOI : https://doi.org/10.4000/yod.339

Ce document a été généré automatiquement le 8 juillet 2021.

\section{(c) (†) 8}

Yod est mis à disposition selon les termes de la Licence Creative Commons Attribution - Pas d'Utilisation Commerciale 4.0 International. 


\section{Le rapport judéo-arabe dans le roman de Abraham B. Yehoshua : La Mariée libérée}

The Jewish-Arab Relationship in the Novel by Abraham B. Yehoshua, The

Liberated Wife ("La Mariée libérée")

יחסי יהודים-ערבים ברומן "הכלה המשוחררת" לא.ב.יהושע

Françoise Saquer-Sabin

\section{Introduction}

1 Le roman d'A.B. Yehoshua traduit en français sous le titre La Mariée libérée ${ }^{1}$, a été écrit entre 1998 et 2001. S'il peut être qualifié, à mes yeux, de roman de l'Autonomie palestinienne, les événements politiques ayant succédé à l'éclatement de la seconde Intifada en septembre 2000 ont sonné le glas de la période de paix relative qui constitue l'arrière-plan historique du roman. Au point qu'à la lumière de la situation au moment de la parution, l'auteur s'est posé la question de l'opportunité de publier². Il nous appartient toutefois d'étudier l'œuvre dans sa dimension littéraire et sous un aspect synchronique.

Dans un article paru dans le journal Ha-'Arets, l'appréciation de la situation politique dans l'espace temporel du roman fait l'objet d'un rapprochement avec l'article d'Amos $\mathrm{Oz}$ « À la lumière du fort azur $»^{3}$. Oz définit la période d'entre-deux-guerres, alors que l'occupation est déjà bien installée, comme une période de repos intermédiaire entre

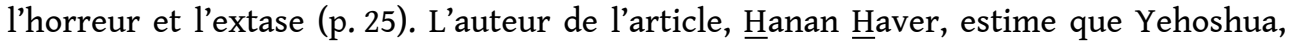
presque trois décennies plus tard, considère la période d'occupation comme une période de repos, une période de non-belligérance plus exactement. Certes, poursuit Haver, le roman n'occulte pas complètement une situation assez éloignée du « repos ». Cependant, la réalité y est représentée de façon édulcorée et rassurante face au fossé insurmontable des contradictions entre la position morale de la société israélienne, et 
le quotidien, peuplé d'actes bien éloignés de la morale dans les territoires occupés, sans parler de la corruption d'un peuple d'occupants.

3 L'auteur de בזכות הנורמאליות(Pour une normalité juive) et l'interlocuteur critique de la position du Palestinien d'Israël Anton Shammas ${ }^{5}$ ne semble plus si sûr de cette normalité dont il se réclamait alors. Il ne paraît pas savoir non plus où mènent les deux axes qui se chevauchent dans le livre : celui qui représente l'illusion de calme occultant les travers de l'occupation, et celui qui témoigne d'une conscience de la crise, sans toutefois y apporter de solution.

4 Sur le plan littéraire, le traitement du personnage de l'Arabe palestinien dans ce roman et la thématique dont ce personnage est porteur rompent radicalement avec toute la littérature qui précède ${ }^{6}$. Pour la première fois, et de façon complète, se produit une égalité de traitement, sur tous les plans, entre les personnages juifs et les personnages arabes. Encore une fois, dans la lignée du traitement du personnage arabe palestinien tout au long $\mathrm{du} \mathrm{xx}^{\mathrm{e}}$ siècle, la définition s'opère à partir d'une structure relationnelle. Seulement cette fois, cette structure s'articule en interaction entre les deux parties, tandis qu'auparavant, elle n'agissait que dans un sens.

5 Sur le plan narratif, la volonté de mimétisme d'une part, le désir d'octroyer une parole vraie au personnage arabe d'autre part, se traduisent par l'introduction, dans le corps du texte, de dialogues entiers en arabe translittéré en caractères hébraïques, et traduits en hébreu en note. Même si l'étude précise de cette parole ne fait pas apparaître d'éléments véritablement significatifs, l'intégration d'un discours direct en langue arabe s'inscrit dans la démarche d'ouverture des frontières qui se manifeste, sous plusieurs aspects, dans le roman.

6 La thématique du roman présente également des éléments novateurs, qui procèdent parfois d'un renversement de valeurs. Le désir d'intégration, par exemple, qui constituait un aspect structurant de la description sociologique et psychologique de l'Arabe à partir des années soixante-dix, s'applique maintenant au personnage juif. Les motivations et implications sont toutefois différentes puisqu'ici il s'agit d'une démarche intellectuelle et non pas d'un tremplin pour une intégration sociale, comme c'était le cas pour le personnage arabe des années soixante-dix. Le désir de pénétrer le domaine de l'autre n'est plus à sens unique et l'intégration n'est plus perçue comme un passage obligé. Ce sont principalement les Juifs qui veulent se fondre dans le milieu arabe, tandis que les Arabes paraissent plus stables à l'intérieur de leurs propres frontières.

7 L'arrière-plan culturel, pour ce qui concerne le milieu juif, témoigne déjà d'une thématique de pénétration dans le domaine arabe. Le personnage focalisateur, Yohanan Rivline $^{7}$, est professeur d'études orientales à l'université de Haïfa, et le milieu juif est essentiellement représenté par la petite communauté d'orientalistes juifs. Le milieu arabe, beaucoup plus diversifié, est représenté par un certain nombre d'identités, tant en Israël que dans les territoires palestiniens.

8 Sami Michaël avait été le premier, dans son roman Refuge ${ }^{8}$, à décrire un milieu arabe complexe et à transporter le lecteur au-delà de la ligne verte ${ }^{9}$, dans les territoires palestiniens. Les rapports entre Palestiniens d'Israël et Palestiniens des territoires et entre chrétiens et musulmans étaient longuement développés, comme dans le roman de Yehoshua. L'évolution, dans le traitement du rapport judéo-arabe, est manifeste. En effet, dans Refuge dont l'intrigue commence au début de la guerre du Kippour, seuls les Arabes passent les frontières, tandis que dans La Mariée libérée, les Juifs aussi passent la 
ligne verte. En outre, ce passage, pour l'Arabe d'Israël, apparait comme une chose naturelle, tandis que dans Refuge, il présentait un risque certain. Il est vrai que Refuge se déroulait en période de guerre, tandis que l'intrigue de La Mariée libérée se situe dans une période d'entre-deux. Mais ce n'est pas la seule différence.

Les évolutions politiques, et surtout la révolte des Pierres, ont amené à une redéfinition des rapports entre Israéliens et Palestiniens des territoires, entre Juifs et Arabes. L'Intifada a très sensiblement modifié l'image du Palestinien, tant chez les Arabes que chez les Juifs d'Israël. Le sentiment d'infériorité des habitants de la rive occidentale et de Gaza a fait place à une fierté de leur part, et à une sorte d'admiration de la part de leurs frères d'Israël. Le rapport est désormais inversé, les Palestiniens d'Israël, qui étaient jusque-là considérés comme nantis, sont méprisés pour leur matérialisme, tandis que les combattants palestiniens apparaissent comme les garants de l'âme palestinienne, comme les bâtisseurs de l'avenir. Chez les intellectuels juifs en Israël, la révolte palestinienne a ouvert la voie à un dialogue. Les Palestiniens sont devenus des partenaires, des individus avec qui il est possible de discuter, de négocier, de faire la guerre, mais aussi d'envisager la paix ${ }^{10}$. L'écrivain S. Yizhar définit la situation dans le quotidien Davar:

"Ce n'est pas du terrorisme, ce ne sont pas des désordres, ce n'est pas de la subversion et ce ne sont pas des émeutes. C'est un peuple qui se lève. Il faut être bouché pour ne pas le voir $»^{11}$.

L'évolution du traitement du personnage arabe fait écho à cette reconnaissance de l'autre en tant qu'interlocuteur, en tant qu'aspirant, en tant qu'individu - en tant que partenaire.

\section{La notion de territoire}

11 La narration s'organise sur le mode du montrer avec focalisation sur le personnage principal, Yohanan Rivline. L'intrigue s'enroule autour de deux aspects, personnel et national, et la relation s'établit selon une structure analogique entre ces deux aspects :

- sur le plan personnel, le lien de Rivline avec sa famille et le besoin de comprendre ce qui s'est passé dans ce qui a été vécu comme un drame familial - le divorce du fils après un an de mariage sans aucune explication et sans signe avant-coureur ;

- sur le plan national, le lien avec les Arabes et la tentative de pénétrer leur univers, de les comprendre.

Le protagoniste essaie de comprendre son identité de père et de Juif, au moyen de la confrontation avec sa famille d'une part, avec les Palestiniens d'autre part. Sa femme, Haguit, magistrate, se montre ouvertement hostile aux démarches qu'effectue son époux en retournant dans l'univers du passé afin de comprendre les raisons du divorce de leur fils. Les relations de ce couple paraissent dominées par la personnalité et le charme de $\mathrm{M}^{\mathrm{me}}$ le Juge, dont Rivline est très amoureux. Cependant, c'est le départ de Haguit pour l'Europe qui permet à Yohanan Rivline de se laisser entraîner dans l'intimité de l'univers arabe et de vivre ce qu'il appelle «L'aventure de l'Autonomie " $^{12}$ (p. $267 \mathrm{~h}, 304 \mathrm{f})^{13}$.

13 L'action se déroule selon trois axes principaux: l'univers familial et professionnel à Haïfa, la pension de famille à Jérusalem, les territoires arabes. 
14 L'univers familial est surtout représenté par les relations de Rivline avec sa femme et par ses monologues intérieurs concernant le fils divorcé demeurant, dans un exil volontaire, à Paris. L'univers professionnel est celui des collègues orientalistes de Rivline et de son ancien maître, Carlo Tedeschi et de sa femme Hannah, orientaliste aussi.

15 La pension de famille des ex beaux-parents du fils divorcé attire Rivline comme un aimant. Il croit que la fréquentation de ce lieu lui permettra de percer le secret de la rupture du lien conjugal de son fils.

Les territoires arabes accueillent Rivline qui se laisse guider dans une sorte de passivité heureuse non dénuée parfois d'agacement devant les ruses de ses hôtes pour le retenir ou négocier quelque monnaie d'échange - le diplôme de Samaher ou l'appui auprès des autorités pour le droit au retour d'une Arabe israélienne mariée à un Palestinien de Cisjordanie.

17 L'ensemble de ces actions tourne autour de la quête de comprendre. Comprendre l'histoire nationale à travers les travaux de recherche de l'orientaliste sur l'Algérie, comprendre l'explosion de terreur qui a bouleversé l'histoire du pays. Comprendre l'histoire de son fils, dont il tente vainement de remonter le cours en explorant la pension de famille et ses occupants. Comprendre l'histoire immédiate en s'immergeant dans l'intimité arabe.

\section{Les lieux arabes}

18 Les lieux arabes vers lesquels mène l'intrigue font toujours l'objet d'une visite juive et d'une rencontre. Le roman s'ouvre sur le mariage de Samaher dans le village arabe musulman situé « aux confins de la patrie » (p. 184 h, 210 f), au nord de la Galilée, non loin de la frontière libanaise. Rivline y reviendra seul et sera accueilli avec tous les honneurs dus à son statut, mais aussi de pourvoyeur du diplôme de fin d'études que Samaher tient à obtenir et qui revêt, de toute évidence, une importance particulière pour l'ensemble du village. Le séjour au village sera l'occasion pour Rivline, l'orientaliste, de se fondre dans l'intimité arabe, mais aussi de se ressourcer et de revenir à un ordre ancien :

"Chez nous, les Arabes, vous serez immergé dans le fleuve du temps véritable» (p. 184 h, $210 \mathrm{f}$ ).

19 Les truchements de l'intrigue, et surtout la volonté de ses compagnons arabes, amènent Rivline conduit par Rashed, le cousin de Samaher, en territoire de l'Autonomie palestinienne. D'abord à Jenine dans un milieu arabe chrétien, puis en compagnie de sa femme et de Hannah, la femme du vieil orientaliste Tedeschi, à Ramallah pour un festival de poésie.

Dans l'Autonomie, on assiste à une sorte de renversement des rôles. Rivline ne s'était pas rendu à Jenine depuis une trentaine d'années, après la guerre des Six Jours. Rashed, l'Arabe d'Israël, parfaitement à l'aise dans son domaine, explique à Rivline le fonctionnement complexe des zones A, B, C (p. $206 \mathrm{~h}, 235 \mathrm{f})$, tandis que les policiers palestiniens au checkpoint plaisantent avec le jeune Arabe et «le Juif, ils le regardent comme s'il n'était rien» (p. $207 \mathrm{~h}, 236 \mathrm{f})$. Sur le plan descriptif, une ancienne conception resurgit, celle de l'harmonie du paysage identifié avec celui des temps bibliques : 
" un important groupe de tentes, près duquel deux grands chevaux broutent

paisiblement» (p. $220 \mathrm{~h}, 251 \mathrm{f})^{14}$.

21 présents-absents sont présentés comme étant des Arabes à qui on a pris le village, et d'après un locuteur arabe, « désorientés, sans repos, ils ne savent pas où se trouve leur lieu véritable et ne laissent pas les autres en paix » (p. $460 \mathrm{~h}, 518 \mathrm{f})^{15}$. Le personnage de Rashed incarne la notion de frontière qui constitue l'un des thèmes fondamentaux du roman. Il bouge sans cesse avec son minibus et permet également aux autres (notamment à Rivline) de franchir les frontières, passage clairement défini comme étant une initiation pour le personnage juif :

«[...] le chauffeur arabe aux yeux de braise, à la fois obligeant et hardi, lui a appris, au cours d'une nuit étrange, qu'il pouvait franchir des frontières et pénétrer au cœur des territoires étrangers, sans rien perdre de ses anciennes amours » (p. 334 h, 379 f).

L'un des enjeux du passage des frontières serait-il de surmonter la peur de connaitre, voire de reconnaître l'autre et, ce faisant, de perdre son intégrité ?

\section{Les lieux juifs}

Les Juifs sont définis à deux reprises en termes de mouvement et d'instabilité : «sans repos, ils décollent, atterrissent - vont devenir fous» (p. 373 h, 422 f). Peut-être parce qu'ils s'imaginent encore qu'ils n'appartiennent pas au Moyen-Orient, qu'ils y sont tombés par erreur (p. 89 h, 103 f). En regard le lieu arabe, présenté comme le retour à un ordre ancien (p. $168 \mathrm{~h}, 193 \mathrm{f}$ ), semble représenter cette stabilité que recherche l'orientaliste. Le village représente une sorte d'entité immuable, à l'inverse du personnage de Rashed qui sous cet aspect du non-lieu au sens propre du terme, s'apparente au Juif dont il devra se séparer pour trouver son propre espace à la fin du roman.

\section{L'Israélien - l'Arabe, le Juif}

Pour la toute première fois dans l'histoire de la littérature hébraïque israélienne, la désignation des personnages arabes et juifs s'organise de façon égalitaire, sur un mode d'appréciation subtil et nouveau de la marque identitaire.

Jusqu'à ce roman, le désignateur " arabe » ou « bédouin », ou autre substitut selon les époques, définissait l'autre, celui qui se distinguait d'une norme implicite - celle de l'identité juive de la citoyenneté israélienne. La connotation du terme "Israélien» sous-entendait «Juif », tandis que l'Arabe, pour être israélien, devait être désigné par une double qualification, «Arabe d'Israël» ou «Arabe israélien» ou "Palestinien d'Israël ». En d'autres termes, la composante pluriethnique de l'État ne se reflétait pas dans la connotation du désignateur "Israélien». Il semblerait que le roman de Yehoshua tende à déstructurer cette connotation normative.

En effet, pour la première fois, face au désignateur " Arabe » apparaît celui de " Juif ». Les deux entités se retrouvent sur un pied d'égalité, chacune représentant alternativement l'autre, celle qui se détache de la norme, selon le terrain où l'on se trouve. Il apparaît en outre que la tentative de casser la norme implicite en matière de frontière amène à n'utiliser le terme israélien qu'en réunissant les deux entités, arabe et juive : «Les deux Israéliens » pour désigner Rashed et Rivline (p. 478 h, 539 f), ou 
alors pour distinguer les Arabes citoyens d'Israël de ceux de l'Autonomie palestinienne, (p. $207 \mathrm{~h}, 236 \mathrm{f}$ ).

Au-delà du désir de traiter sur un pied d'égalité les personnages juifs et arabes se profile un glissement de définition identitaire. Reportons-nous à une discussion entre Rivline et son beau-frère qui vit en diaspora :

"Je veux dire qu'il faut rendre à l'israélisme ce je-ne-sais-quoi qu'il y avait dans le judaisme...

[Rivline] - Mais enfin, de quoi parles-tu ?

- Je me demande comment délivrer un peu l'israélisme de son carcan d'attachement au sol, à la terre. Lui redonner des ailes. Essayer d'en extraire quelque chose de plus spirituel pour le monde entier [...] Relier la capacité juive de s'abstraire des contingences historiques aux réussites scientifiques israéliennes, à la curiosité et au pragmatisme, à notre solidarité collective [...] » (p. $453 \mathrm{~h}, 509 \mathrm{f})$.

L'utilisation du désignateur "Juif» face à celui d'«Arabe » témoigne à la fois d'une égalité de traitement littéraire entre les deux entités, et du déplacement d'une définition identitaire " (juive) israélienne» vers une identité plus "juive». Ce qui reviendrait à octroyer aux Arabes une place citoyenne moins marginale dans l'État d'Israël. En même temps, et parce que les deux points de vue se chevauchent comme dans la discussion citée plus haut avec le beau-frère, celui de l'israélienneté et celui de la judéité, le désignateur «État juif » (p. 433 h, 487 f) ou « État des Juifs » (p. 511 h, 577 f) apparaît, mais à travers une conscience énonciatrice arabe.

\section{La définition identitaire}

\section{Identification - Autonomisation}

Deux personnages masculins périphériques fonctionnent comme moteurs de l'intrigue et représentent chacun, pour le personnage principal, Rivline, la figure du père et celle du fils :

le père spirituel de Rivline, le vieil orientaliste, Tedeschi, le jeune Arabe, Rashed, qui lui rappellent ses deux fils (p. 203 h, 232 f).

Les figures féminines présentent également un certain nombre d'identifications autour du désignateur kalah, traduit par "mariée", mais qui signifie aussi "fiancée", «brue » et peut aussi désigner affectueusement la femme aimée. C'est ainsi que s'accordent autour de cette désignation les deux jeunes femmes :

- Samaher, l'étudiante arabe de Rivline qui invite toute la section d'études orientales à son mariage, et dont les atermoiements et exigences constituent l'un des moteurs de l'intrigue ;

- Galia, l'ex-femme du fils cadet du couple Rivline qui n'a jamais compris ni accepté son départ de la famille.

Deux femmes d'âge mur se rejoignent aussi autour de la notion de kalah, dans le sens affectif :

- Haguit, la femme de Rivline, magistrate de profession, à la fois très présente, peut-être trop présente et directive dans la vie de son mari, et absente puisque son départ pour un procès en Europe permet à Rivline de vivre l'aventure arabe ou comme il l'appelle «l'aventure de l'Autonomie » (p. 267 h, 304 f)).

- Afifa, la mère de Samaher, qui fut l'étudiante de Rivline autrefois, et dont le charme et la beauté ne cessent d'attendrir et d'attirer ce dernier. 

descriptions. Fouad, le serviteur arabe, le majordome de la pension de famille des ex beaux-parents du fils Rivline, présente de forts éléments d'identification avec Yohanan Rivline. Leur communauté d'âge et la mention de leur chevelure argentée sont fréquemment énoncées en termes identiques (notamment, pp. 324, 429 h, 368, 483 f). La complicité et l'amitié entre les deux hommes sont portées par les désignations appliquées à Fouad qui passent du champ sémantique de la fonction de serviteur (employé, majordome, etc.) à celui de partenaire (associé, guide, etc.). Au fur et à mesure qu'évolue la quête de Rivline en vue de comprendre ce qui s'est passé au sein de l'ex-belle-famille de son fils pour aboutir au divorce de ce dernier, Fouad apparait comme étant le gardien du secret.

Il apparait alors que, dans la dynamique de l'action, un rapport inversement proportionnel s'établit entre Rivline d'une part, Fouad et Rashed, d'autre part. À mesure que le protagoniste juif est emporté, bon gré mal gré, vers l'intimité des différents milieux arabes dans le minibus de Rashed, à mesure qu'il se laisse absorber par une volonté autre, parfois vécue par lui comme contraignante et insidieuse, les personnages de Fouad et de Rashed se développent et s'autonomisent. Le personnage de Fouad prend de l'ampleur, passe de l'état de serviteur fidèle limité à la sphère familiale dont il détient toutes les clés, à celui d'homme libre, et même de poète. Au point qu'il démissionne à la fin, procédant ainsi à une redéfinition de son personnage.

personnage de Rashed se déploie aussi dans toutes ses dimensions et remplit de multiples rôles - chauffeur, émissaire, intermédiaire, amoureux sans espoir de sa cousine, mais aussi sorcier et enfin présent-absent. Il incarne la notion de frontières qu'il traverse sans cesse dans tous les sens, en entraînant parfois les Juifs et particulièrement Rivline avec lui, mais c'est par la séparation qu'il trouvera sa voie et sa stabilité.

Fouad, plus âgé et plus sage, définit d'emblée ses limites à l'intérieur des frontières :

"Justement, un homme comme moi, membre d'une minorité, à qui on a donné toutes les clés de l'hôtel, doit veiller tout particulièrement à ne pas s'empêtrer dans les frontières des autres » (p. 344 h, $391 \mathrm{f})$.

Le personnage juif de Rivline est désigné et décrit, lorsqu'il est confronté au milieu arabe, dans des termes qui mettent en évidence son statut hiérarchique («Professeur »), mais aussi sa position à la limite entre deux frontières, caractérisée par la double désignation «orientaliste juif». Au village ou dans les territoires de l'Autonomie, il est « le Juif» ou « l'invité juif ». Il est piquant de noter le renversement des rôles, la notion d'invité qui a été véhiculée par tant de personnages arabes dans des textes narratifs antérieurs, y compris chez Yehoshua ${ }^{16}$, s'applique ici au personnage juif.

37 L'imbrication des différents niveaux de l'intrigue fait également apparaître des analogies. Le roman s'ouvre sur un mariage, celui de l'étudiante arabe Samaher. Or, le mariage et sa dissolution constituent le noyau de l'intrigue principale, et c'est en raison du mariage raté de son fils que Rivline ne supporte plus ce type de cérémonie. Bien qu'il s'agisse d'un mariage arabe, ce qui semble constituer, aux yeux du protagoniste, une distance de sécurité affective, le serpent de la jalousie se réveille (p. $9 \mathrm{~h}, 11 \mathrm{f}$ ) et l'identification commence entre le mariage de Samaher et celui de son fils, entre Samaher et Galia, la brue tant regrettée. Autre analogie, si le mariage de son fils s'est terminé par un désastre, celui de Samaher ne semble pas se réaliser dans la joie. La 
jeune mariée succombe vite à une profonde dépression et le mari est qualifié de "triste ", à l'instar du fils Rivline, Ofer, inconsolable, que son père supplie, dans une fausse interlocution : " ne sois pas si sombre et si triste» (p. 221 h, 252 f). Dans les deux cas, la jeune mariée sombre dans un mutisme qui ferme la communication avec son époux. Mariages ratés et nostalgies amoureuses semblent être le lot des jeunes hommes. Ofer, le fils Rivline, s'entend reprocher par Fouad de vouloir ranimer la flamme d'un amour perdu, de même que Rashed se consume pour sa cousine nouvellement mariée (p. 538 h, 611 f). Le mari sans nom de Samaher semble, quant à lui, extérieur à l'histoire.

La recherche de l'orientaliste sur l'Algérie et les violences qui s'y déchaînent est mise en parallèle avec la situation intime de Yohanan Rivline. Il ne comprend pas plus comment cette violence a pu exploser, alors qu'il se révèle incapable d'en identifier les signes avant-coureurs, qu'il n'explique le divorce de son fils sans raison apparente.

\section{Identité - Altérité}

39 Sur le plan personnel, la désignation et la description font une large part au désir de sympathie, de rapprochement, d'intimité dont témoigne l'instance énonciatrice, et qui s'exprime en général par le personnage de Rivline. L'orientaliste éprouve une sympathie grandissante pour Rashed qui lui rappelle ses deux fils, et pour Samaher dont l'intelligence et le sens de la langue hébraïque forcent son admiration. Il est également fortement ému par la belle femme pleine de charme qu'est la mère de Samaher. Une amitié profonde et une véritable complicité le lient à Fouad, l'homme de confiance de l'ex beau-père de son fils.

Cependant, l'étude du schéma relationnel se conclut sur une impression de duperie. Dès le départ, la relation s'établit sur une sorte de marchandage plus ou moins implicite.

41 L'univers des orientalistes est présenté, avec ses intrigues et rivalités universitaires, à travers trois types de caractères. Rivline selon les termes de ses amis arabes est un « invité juif très respectable, et qui sait l'arabe » (p. 200 h, 229 f). Akri, le nouveau responsable du département, lui-même d'origine orientale, parle un arabe parfait et pas seulement livresque, contrairement à Rivline. Cet homme religieux oppose à l'empathie que manifeste Rivline vis-à-vis des Arabes et à son désir de les connaitre de l'intérieur, une attitude qui peut paraitre cynique, mais que les évolutions et le dénouement du roman définissent plutôt comme réalistes :

« Nous devons étudier les caractéristiques des Arabes pour être sur nos gardes. [...] Savoir à quoi s'attendre de leur part et ce qu'il ne faut pas espérer. Respecter ce qui leur est réellement précieux, comprendre ce qui leur fait mal, pour se garder à bon escient des trahisons et des mensonges. » (p. 94 h, 108-109 f).

Pour Akri, l'ouverture aux Arabes est absolument nécessaire, mais en aucune façon l'intimité, qui ne saurait se solder que par l'incompréhension (p. 272 h, 310 f). Tedeschi, le maître, qui avait formé tant de générations de chercheurs et d'érudits, proclamait: « Nous n'avons aucun espoir de comprendre rationnellement les Arabes, et il ne nous reste plus qu'à revenir à l'étude de leur poésie » (p. 500 h, p. 565 f). C'est ce que fait sa jeune femme, une de ses anciennes élèves, qui lui a consacré sa vie avec dévotion. Hannah, l'orientaliste qui traduit de façon magistrale la poésie préislamique offre peut-être le seul moment de dialogue véritable et signifiant dans un duo arabehébreu, lors du festival de poésie à Ramallah. Un échange poétique poignant lorsque Le Poète palestinien, clairement identifié à Mahmud Darwish même s'il n'est pas nommé, 
lit de la poésie arabe ancienne et que Hannah lui fait écho en traduisant directement en hébreu. C'est le seul moment où l'introduction de la langue arabe est significative. cousine alitée. Il véhicule Rivline dans différents lieux arabes et lui fait passer les frontières. C'est au cours de leur voyage en Cisjordanie qu'il confie, plus ou moins explicitement, à son passager la mission d'user de son influence pour que sa sœur, mariée en Cisjordanie, puisse revenir en Israël. La tonalité du schéma relationnel opère un brusque changement à partir du moment où Rashed s'aperçoit que Rivline n'est pas à même de réaliser cette mission. Parallèlement, la position de demandeur de la famille de Samaher fait place à une attitude d'exigence (p. 548 h, 621 f). Rivline, de son côté, face à l'échec de sa quête personnelle concernant le divorce de son fils, renonce à l'espoir d'obtenir de l'aide de la part des Arabes (p. 502 h, 567 f). 
frontière dans le roman. La position inconfortable de l'orientaliste israélien est qualifiée de double piège :

- il est suspecté de pessimisme, car se trouvant en guerre avec son sujet d'étude ;

- mais taxé d'optimisme en raison de son désir de paix et de conciliation.

Tedeschi avait senti le piège et pris garde de préserver une distance, d'éviter la symbiose avec les Arabes. En effet, explique Rivline, pour les Israéliens, l'orientalisme ne fait pas l'objet d'une simple recherche scientifique, mais de questions qui touchent à leur avenir, de questions de vie ou de mort :

"Ils doivent se garder de toute expression de mépris, de mésestime, de condescendance à l'égard de leurs voisins, qui sont également l'objet de leur recherche. Et d'autre part, ils doivent tout autant éviter une attitude de mansuétude, de bienveillance romantique. Les Israéliens sont des voisins proches, immédiats, des otages. Qu'ils le veuillent ou non, ils font intégralement partie de l'objet de leur investigation. » (p. 499 h, 563 f).

L'illustration concrète de cet exposé théorique est apportée par la mort dans un attentat d'un chercheur orientaliste, un Juif religieux orthodoxe, qui a consacré sa vie à étudier la littérature populaire arabe, et notamment algérienne. Ce sont les textes qu'il a étudiés que Rivline souhaite exploiter pour sa recherche, mais ils sont écrits dans un arabe trop populaire pour lui, et il demande à Samaher de les lui traduire. Travail qui pourrait être pris en compte pour valider le diplôme tant attendu. La chaîne d'interdépendance est bouclée.

\section{La distanciation}

51 Le procédé de distanciation se cristallise dans deux domaines - le théâtre et la recherche.

\section{Les travaux de recherche sur l'Algérie}

52 La recherche de Rivline sur l'Algérie apparaît comme un moyen détourné de traiter du problème arabe sans attaquer de front le conflit israélo-palestinien. Au moyen d'une distanciation déjà éprouvée par l'auteur sur d'autres sujets brûlants ${ }^{17}$, le parallèle avec la violence déchaînée en Algérie permet une réflexion sur certains sujets, par exemple les questions d'identité (p. $264 \mathrm{~h}, 300 \mathrm{f}$ ), de langue (pp. 356-357 h, 404-405 f), de collaboration à travers le problème des Harkis (p. 226 h, 257 f). Ces questions sont traitées en général à travers la conscience du personnage de Rivline qui fait le point sur l'état de ses recherches. Toutes sont susceptibles de s'appliquer au problème palestinien.

Un autre aspect, d'ordre affectif cette fois, apparaît au détour du problème algérien. L'orientaliste au grand cœur dévoile certains sentiments que, dans son quotidien pétri de bonnes intentions, il ne se permettrait pas d'exprimer ni même de ressentir. La distanciation permet de mettre à jour, dès le début du roman, ce que les actes du protagoniste s'efforcent de contredire. Rivline s'avoue déçu de tous les Arabes et ajoute :

«Comment peut-on écrire avec la moindre sympathie sur la lutte algérienne des années 1940-1950, lorsqu'on voit les terribles massacres et le terrorisme dément qui sévit là-bas aujourd'hui » (p. $37 \mathrm{~h}, 45 \mathrm{f})$.

54 Son propos a beau se concentrer sur l'Algérie, la déception s'applique bien à « tous » les Arabes. Le parallèle avec la lutte palestinienne est amené par l'énoncé d'un locuteur 
palestinien qui compare la situation des Arabes d'Algérie oppressés par les français à celle des Palestiniens : «Mais quand allez-vous écrire sur nous, Professeur Rivline?». Rivline explique alors qu'en écrivant sur les Arabes lointains, il cherche à se rapprocher du récent, de l'actuel. «Car vous êtes en fin de compte tous de la même origine, du même désert » (p. 192 h, 219 f).

Finalement, à la lumière de ces parallèles et surtout au vu de la déception annoncée d'emblée par le personnage qui manifeste pourtant le plus de sympathie à l'égard des Arabes, le processus de séparation finale était annoncé. Dans cette perspective, l'ensemble du schéma relationnel évolue en mouvements concentriques pour revenir à un présupposé. Toute la dynamique de ce mouvement, comme nous l'avons évoqué plus haut, est impulsée par la quête de chacun et le besoin qu'il a de l'autre pour la satisfaire. Une fois l'espoir déçu concernant l'aide attendue, la boucle se ferme sur un état d'esprit initial plus ou moins énoncé en filigrane.

\section{Le théâtre}

La plus belle illustration du rapport identité-altérité traité de façon distanciée est offerte par le biais du théâtre palestinien. Et surtout, les séquences liées au théâtre ou à la poésie constituent, sans conteste, les plus beaux moments du roman. Prestations savoureuses qui concentrent, comme dans une mise en abyme, la thématique identitaire du roman, et se situent toutes en lieu arabe.

L'aventure de l'Autonomie amène Rivline, d'abord seul puis avec sa femme, à assister à deux manifestations culturelles à la fois étranges et émouvantes. Son premier voyage, un peu forcé, à Ramallah en compagnie du jeune Rashed l'introduit dans un milieu arabe chrétien où le récital d'une jeune libanaise chrétienne orthodoxe le bouleverse autant qu'une pièce de théâtre qu'il a vue avec sa femme et qui reproduisait des scènes de la Genèse. Mais c'est le spectacle de deux jeunes séminaristes diplômés du Séminaire orthodoxe d'Athènes, d'où leur surnom Socrate et Platon, qui incarne la dualité identité-altérité. Les deux chansonniers miment une scène du tribunal et remplissent alternativement les rôles de l'Israélien et du Palestinien. Jusqu'à ce que les deux se fondent en un seul personnage à la fois misérable et monstrueux qui balbutie et se lamente dans les deux langues, se frappe, se caresse, sanglote et rit (p. 223 h, 254 f). Plus tard, la scène du dibbouk jouée par Samaher et son cousin Rashed émeut profondément le public juif qui s'exprime par la voix de Rivline : «Je n'arrive pas à croire ce que vous êtes capable de faire lorsque vous vous identifiez à nous » (p. 435 h, 489 f).

\section{Autonomisation}

La narration extradégiétique ou récit du il fait parfois place, de façon sporadique, à un autre type de narration.

Une fausse interlocution s'appliquant au personnage de Rivline se produit dans des situations où ce personnage est seul avec lui-même, dans une situation émotionnelle insolite et forte. De façon significative, la première manifestation de ce passage du il au tu s'opère lors d'une insomnie, juste avant que sa femme ne parte pour quelques jours en Europe pour des motifs professionnels. Or, le départ de sa femme marque le début de ce que Rivline appelle "l'aventure de l'Autonomie ». Autonomie palestinienne, certes, mais aussi autonomie personnelle lorsque, en raison de l'absence de sa femme, il se 
trouve livré à lui-même, sans contrainte extérieure. C'est là que commence ce voyage un peu fou qu'il ne domine pas, au-delà des frontières arabes jusqu'au village de Galilée, puis à Jenine où il passera la nuit. Et la dernière fois qu'une fausse interlocution appliquée au personnage de Rivline casse le rythme du récit, c'est lorsque son ex bellefille, Galia, fait irruption dans son cours à l'université, visite qui marque le point de départ du dénouement concernant l'intrigue personnelle et familiale du roman.

Entre ces deux moments narratifs, les quelques fois où un narrateur extradiégétique s'adresse directement à Rivline, ce phénomène constitue une pénétration dans l'intimité profonde du personnage momentanément libéré de contraintes extérieures, et toujours lorsqu'il se trouve en milieu arabe (au village, un soir de ramadan, et à Jenine). L'intériorité du personnage se révèle alors dans une sorte de complicité avec le lecteur, qui passe du statut de témoin à celui de partenaire, presque de coénonciateur, comme si l'interlocution qui n'identifie qu'un seul locuteur laissait une place ouverte à son face-à-face.

61 En ce sens, la narration participe pleinement à la notion de passage des frontières. D'autant que ce passage ne peut vraisemblablement se faire que dans une situation d'autonomie. L'absence de l'épouse donne à Rivline une liberté d'action et de pensée qui se révèle, sur le plan narratif, par le passage du il au tu, de la "non-personne » à une véritable personne du dialogue ${ }^{18}$. Cette forme d'autonomisation du personnage va de pair avec celle du personnage arabe, et particulièrement celui de Rashed.

Comme nous l'avons mentionné précédemment, l'évolution du schéma relationnel est marquée par une mutation irréversible, dominée par la déception et la rancœur lorsque les protagonistes comprennent que l'utilisation de l'autre, dans la réalisation de leur quête, demeure infructueuse. À partir de ce moment, le personnage de Rashed, caractérisé et défini par ses proches eux-mêmes comme instable et dérangeant, en raison de son statut de présent-absent, ce personnage qui incarnait la notion de frontière, prend ses marques et se détache du Juif. Le processus d'autonomisation psychologique et affective est relayé, comme pour le cas du personnage juif, par une rupture du modèle narratif. La narration sur le mode du montrer fait place à une fausse interlocution, et le passage du il au $t u^{19}$ remplit, dans ce cas, une fonction de séparation doublée d'une affirmation identitaire. Le personnage de Rashed se détache de la perspective imposée par le personnage juif. Pour la première fois, la focalisation ne passe pas par le personnage de Rivline, la scène se déroule hors de sa présence et hors de sa conscience. Le personnage arabe s'autonomise et marque son espace représenté, dans un rapport métonymique, par la chambre qu'il occupe au village. Il vide sa chambre pour y accueillir sa sœur cisjordanienne dont il prend le destin en mains et secoue le matelas sur lequel a dormi le Juif Rivline. Le mot זכות, « droit » apparaît sept fois dans le chapitre pour s'actualiser dans le droit au retour, זכות השיבה. Sur le plan affectif, il renonce, en quittant le village, à la proximité avec sa cousine, ce qui signifie aussi ne plus convoiter le territoire d'autrui. Le passage des frontières, tel qu'il le pratiquait de façon frénétique, est dès lors présenté comme une utopie, tandis que la réalité est énoncée en termes de "droit » et de «loi ». Il s'agit de "passer du stade d'Israéliens virtuels à celui d'Israéliens réels» (p. 510 h, 577 f). Le véritable droit à l'autonomie ne peut se faire qu'au prix d'un processus de séparation.

63 Le dénouement de l'intrigue, pour ce qui concerne l'aspect national et le rapport avec les Arabes, place le passage "du stade d'Israéliens virtuels à celui d'Israéliens réels » dans un contexte dramatique qui frise l'humour noir. Le phénomène d'autonomisation 
du personnage arabe est marqué par un bref passage narratif au discours du je appliqué au neveu de Rashed, égaré dans la zone frontalière. L'épisode apparaît comme une sorte de fable où alternent le monologue intérieur du petit garçon et les dialogues de deux druzes qui chassent le sanglier. Une balle perdue atteint l'enfant qui se retrouve en effet en Israël, mais en état de mort clinique. Le petit Arabe, en quête de citoyenneté israélienne, qui voulait devenir un Israélien réel, a perdu conscience et vie réelle tout en acquérant le droit au retour. En effet, il avait été précisé que les neveux de Rashed pourraient rejoindre leur mère en Israël s'ils étaient atteints d'une grave maladie.

Le décryptage des mutations narratives évoquées fait clairement apparaître un processus de séparation, une prise de conscience de la nécessité de définir ses propres marques quitte à renoncer au rêve et à l'illusion pour mieux se battre avec la réalité. Toutefois, l'autonomisation, d'après le dénouement ironico-tragique, ne paraît pas viable.

\section{Conclusion}

Le schéma évolutif du roman, sous ses différents aspects, narratif et thématique, fait apparaitre en fin de parcours une position idéologique complexe autour de la notion de frontière. Après avoir développé de multiples vecteurs véhiculant des notions d'échange, d'ouverture, d'interpénétration, le roman se conclut sur les antonymes de ces mêmes notions - séparation, cloisonnement, unilatéralité.

$\mathrm{Si}$, encore une fois, le personnage arabe prend ses marques en relation avec le personnage juif, il s'autonomise toutefois sur le plan littéraire. Le personnage de Rashed incarne cette autonomie littéraire que ses prédécesseurs ont tardé à acquérir. L'aspect thématique conclut sur une déception et une violence de sentiments à l'égard du Juif qui n'a pas répondu aux attentes de la famille. Mais malgré tout, l'évolution des personnages arabes, comme Samaher, sa mère ou Rashed se traduit par une affirmation de soi et de ses droits qui n'est pas présentée comme négative. Le renoncement à l'autre est perçu comme une autonomisation plus que comme une défaite.

L'évolution du personnage de Rivline n'est pas similaire à celle des personnages arabes, et particulièrement à celle de Rashed. Le personnage juif n'était pas en recherche d'identité, mais plutôt d'empathie et de compréhension. Sa pénétration dans le territoire de l'autre procédait d'un désir de perfectionnement, de complément de formation, plus que d'un besoin identitaire, même si ce désir d'intimité était en relation avec sa recherche familiale et personnelle. Le processus de séparation, dans son cas, justifie, en quelque sorte, les opinions de ses collègues qui avaient, dès le départ, défini des frontières et dénoncé les dangers de rentrer dans l'intimité des Arabes.

L'évolution du personnage juif est finalement moins inattendue que celle $d u$ personnage arabe. Il retourne à un certain ordre logique et préétabli après s'être égaré dans des voies quelque peu audacieuses. Le personnage arabe, en revanche, qu'il s'agisse de Rashed ou de Fouad, suit un parcours véritablement autonome et se distingue de la situation initiale qui était la sienne.

La portée idéologique du roman, véhiculée en grande partie par le personnage de Rivline et par ses tentatives de comprendre l'autre, atteint son paroxysme dans la découverte du motif ayant entraîné le divorce du couple du jeune Rivline. Le secret de l'inceste qui, à première lecture peut paraître artificiel et déplacé, symbolise de façon 
générale le tabou et l'effondrement de la tribu. Si le personnage de Rivline incarne le doute, le tourment et la recherche de l'autre, la découverte de l'inceste lié, même indirectement, à l'histoire personnelle de son fils, symbolise la brisure de l'intégrité israélienne qui se reflète dans différentes strates de l'organisation du roman.

\section{BIBLIOGRAPHIE}

GROSSMAN, David (8 décembre 1989), « ארץ רעולת פנים («Une terre au visage voilé »), in Yedi'ot 'Aharonot.

GROSSMAN, David (1998 [1992]), Les Exilés de la Terre promise (נוכחים נפקדים), Éditions du seuil, Paris.

MAINGUENEAU, Dominique (1988 [1985]), Éléments de linguistique pour le texte littéraire, Bordas, Paris.

MICHAEL, Sami (1977, en hébreu), חסות (Refuge), 'Am ‘Oved, Tel-Aviv.

SAQUER-SABIN, Françoise (2002), Le personnage de l'Arabe palestinien dans la littérature du XXe siècle, CNRS Éditions.

SMILANSKI, Yizhar (1956, 1998 [1948]), « Le prisonnier » ( השבוי »), in Pages israéliennes, Pierre Seghers éditeurs, Paris 1956 (trad. Joseph Milbauer) et « Le Prisonnier », in Anthologie de nouvelles israéliennes contemporaines, Gallimard, Paris, 1998 (trad. Laurent Schuman)

YEHOSHUA, Avraham B. (1992 [1980]), Pour une normalité juive (בזכות הנורמאליות), Éditions Liana Lévi, Paris. (trad. Eglal Errera et Amit Rotbard).

YEHOSHUA, Abraham B. (2001), La mariée libérée (המשחררתהכלה), Calmann-Lévy, Paris. (trad. Francine Levy).

\section{NOTES}

1. Abraham B. Yehoshua, הכלה המשחררת (hébreu, 2001), La Mariée libérée, Paris, Calmann-Lévy, 2001 (trad. Francine Levy). Le sens exact du titre hébraïque est La fiancée ou la mariée «libératrice». Les citations, pour certaines, sont extraites de cette édition, d'autres sont effectuées par mes soins dans un souci de fidélité à l'original. Dans tous les cas, la référence du texte français est mentionnée.

2. Abraham B. Yehoshua s'en est expliqué, notamment lors d'un entretien sur France Culture en octobre 2003.

3. L'article de 'Oz, écrit en 1972, a été compilé dans un recueil éponyme באור התכלת העזה: la lumière d'un fort azur), Sifriat po'alim, 1979. Quant au rapprochement avec le roman de Yehoshua, il figure dans l'article de $\underline{H} a n a n$ Haver, "הקלות המשחררת 》 (La légèreté libératrice), in Sefarim, Musaf ha-'Arets, 10.10.2001.

4. Voir Yehoshua, Abraham B. (1992[1980]). 
5. A.B. Yehoshua a mené un débat public avec l'auteur palestinien de nationalité israélienne, Anton Shammas, sur la question de l'identité israélienne. Voir : Saquer-Sabin, Françoise (2002), pp. 55-56.

6. Cf. Saquer-Sabin, op. cit.

7. L'orthographe des noms propres s'aligne sur la traduction française.

8. Voir : Michaël, Sami (1977). Cf. Saquer-Sabin, op. cit., pp. 45-48, 103-105, 109-118.

9. On désigne par «ligne verte» la délimitation entre le territoire d'Israël et les territoires conquis lors de la guerre des Six Jours en 1967.

10. L'écrivain David Grossman développe cette idée dans un article publié dans le quotidien Yedi'ot 'Aharonot le 8 décembre 1989 sous le titre: "ארץ רעולת פנים", "Une terre au visage voilé ».

11. Davar, 30 décembre 1987.

12. La traduction française donne "l'aventure autonomienne", je préfère "l'aventure de l'Autonomie » qui préserve la polysémie entre l'Autonomie palestinienne et l'autonomie accordée à Rivline par l'absence de sa femme.

13. Les références apporteront, sur ce modèle, d'abord le texte original hébraïque $(h)$, puis la traduction française dont les références figurent à la note 1 (f).

14. Dans la nouvelle de S. Yizhar, "Le Prisonnier », le paysage, identifié aux sources bibliques, apparaît comme idyllique avant l'arrivée des soldats israéliens : Smilanski Yizhar, השבוי (hébreu, 1948), pp. 111-112.

15. La notion de Présent-Absent fait l'objet d'un essai éponyme de David Grossman, voir: נוכחים נפקדים, Présent-Absent (hébreu, 1992), p. 64 ou en français Les Exilés de la terre promise (trad. Katherine Werchowski), p. 70.

16. Dans son roman'Amant (hébreu, 1979) ; mais aussi dans תוסמאהבRefuge de Sami Michaël (hébreu, 1977), Chambre close de Shimon Ballas (hébreu, 1980), טוב ערבי Bon Arabe de Yoram Kaniuk (hébreu, 1984), חרוד לעין הדרך La Route d'Ein Harod de Amos Kenan (hébreu, 1984).

17. Abraham B. Yehoshua avait écrit le roman מסע אל תום האלף, Voyage vers l'an mil (hébreu, 1997) en décalant dans le temps le problème des relations ashkénazes-séfarades. מר מאנ Monsieur Mani (hébreu, 1990) témoigne également de cette démarche.

18. Rappelons que « la délimitation de la classe des embrayeurs a pour effet de dissocier le couple $j e-t u$, véritables «personnes» du dialogue, du pronom il, véritable pro-nom, que Benveniste préfère placer dans le registre de ce qu'il appelle la non-personne, celui des objets du monde, autres que les interlocuteurs. » Voir : Maingueneau, Dominique (1988[1985]), p. 6.

19. Il s'agit du chapitre 16 de la dernière partie intitulée Shihrur, Délivrance.

\section{RÉSUMÉS}

Ce roman particulièrement « imprégné » d'autonomie palestinienne, La mariée libérée présente un aperçu très original de l'expression de relations judéo-arabes en Israël. Pour la première fois, le choix littéraire met les personnages juifs et arabes sur un pied d'égalité. Par exemple, dans la partie principale du texte, des dialogues en arabe (traduit dans les notes de bas de page en hébreu) s'efforcent de rendre les véritables paroles des Palestiniens. L'interaction entre les deux parties varie aussi d'après les thèmes, avec une alternance des rôles du dominant et du dominé selon les lieux et les territoires. Toutefois, traverser les frontières n'est pas sans danger pour 
l'intégrité de chaque personne, même si c'est un moyen de briser le mur des malentendus, et la conclusion en serait l'effondrement symbolique de l'identité nationale.

A novel definitely steeped in Palestinian autonomy, "La mariée libérée" presents very original insights into the expression of Jewish-Arab relationships in Israel. For the first time, the literary choices put Jewish and Arab characters on an equal footing. For example, in the main part of the text, dialogues in Arabic (translated in footnotes into Hebrew) strive to give the true spoken words of Palestinians. The interaction between the two parties is also at play trough the themes with alternating roles of the dominator and dominated according to the geographical territories. However, crossing the boundaries, is not without danger for each person's integrity even if it breaks the wall of misunderstandings, and the conclusion is the symbolic collapse of national identity.

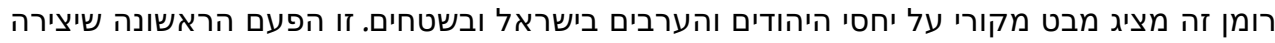
ישראלית מציגה אותם על מישור אחד, כאשר, כדוגמה, הטקסט כולי מביל קטעים ארים מרוכים בעים בערבית

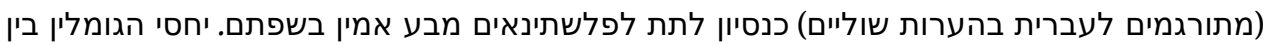

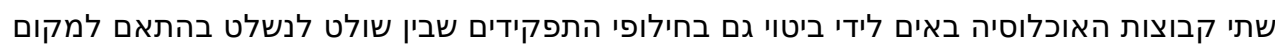

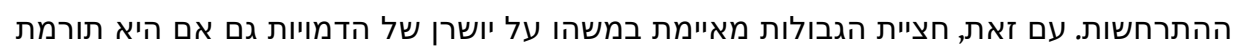

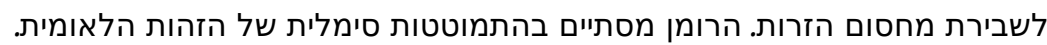

\section{INDEX}

Keywords : Arabs and Israelis, Israeli novel, Israeli-Palestinian conflict, Israeli-Palestinian relations, literature, twentieth century, Yehoshua Abraham B. (1936-)

Mots-clés : arabes et Israéliens, conflit israélo-palestinien, relations israélo-palestiniennes, roman israélien, Yehoshua Abraham B. (1936-)

Thèmes : littérature

\section{מילות מפתח}

אברהם ב יהושע, המאה העשרים, ישראל, ספרות, ערבים וישראלים:

Index géographique : Israël

Index chronologique : vingtième siècle 